Furthermore, IFN-related mediators might be more easily applicable biomarkers of IFN upregulation. ${ }^{4}$

Material and methods Twenty-three iSLE patients (ANA titer $\geq 1: 80$, symptoms $<5$ years, $\geq 1$ objectified clinical ACR criterium), 25 quiescent SLE patients (fulfilling ACR criteria, SLEDAI $\leq 4)$ and 11 healthy controls were included.

The IFN score was determined in monocytes, based on 14 IFN-related transcripts, representing all three IFN-modules. (M1.2: CXCL10, IFI44L, IFIT3, LY6E, MX1 and SERPING1. M3.4: AIM2, IFITM1, IRF7 and STAT1. M5.12: C1QA, CSCL2, IFI16 and IRF9). IFN-scores $>95$ th percentile of controls were defined as positive.

Levels of IFN-related mediators, including IFN- $\gamma$ induced protein 10 (IP-10), monocyte chemo-attractant protein (MCP$1)$, and Myxovirus-resistance protein $\mathrm{A}(\mathrm{MxA})$ were measured using ELISA.

Results IFN-score was increased in 52\% of iSLE patients and $48 \%$ of SLE patients. Of iSLE patients, 52\%, 52\% and 48\% respectively had upregulation of M1.2, M 3.4 and M5.12 (see figure 1). In SLE patients, respectively 52\%, $44 \%$ and $40 \%$ were upregulated. M3.4 and M5.12 were only upregulated if M1.2 was activated.

Both MxA and IP-10 were increased in iSLE (median $120 \mathrm{ng} / \mathrm{ml}$ and $76 \mathrm{pg} / \mathrm{ml}$, respectively) compared with controls (median $82 \mathrm{pg} / \mathrm{ml}$ and $23 \mathrm{pg} / \mathrm{ml}$, respectively). MxA and IP-10 did not correlate in iSLE. In SLE, MxA was increased (median $111 \mathrm{ng} / \mathrm{ml}$ ), while IP-10 was not. MCP-1 levels were not significantly different between the groups.

Levels of MxA correlated with IFN-score in both iSLE $(r=0.49, p=0.0171)$ and SLE $(r=0.70, p<0.0001)$. Levels of IP-10 correlated with IFN-score based on the 14 genes in SLE but not iSLE.

Conclusion IFN-signature is present in 52\% of iSLE patients and correlates with MxA. We hypothesise that these patients might be at risk for disease progression. Longitudinal data however should be awaited. Interestingly, MxA levels correlated strongly with IFN-score and thus could be a suitable and easily applicable surrogate marker.

\section{PS1:6 DIFFERENTIAL DIAGNOSIS OF AUTOIMMUNE DISEASES, OUTLIER DETECTION PLUS SUBGROUPING IN CLINICAL TRIALS BY HIGH CONTENT AUTOANTIBODY PROFILING}

P Schulz-Knappe, P Budde, H-D Zucht. Protagen AG, Dortmund, Germany

10.1136/lupus-2018-abstract.55

Purpose Early diagnosis as well as initiation of successful treatment are two big challenges in the management of patients with autoimmune diseases (AID). Overlap of a plethora of clinical symptoms, ranging from multi-organ involvement, fatique, inflammation to CNS-involvement make differential diagnosis quite challenging. Especially in early disease these signs are difficult to quantify, hence the lag time from start of disease until clinical diagnosis may be delayed, sometimes for years. With the growing interest in conducting clinical trials in AID, there is a need for new biomarkers that can be used to diagnose individual AIDs to reduce the inclusion of patients not carrying the intended disease, and identify clinical subsets, predict treatment outcome and assess disease activity.
Methods The autoantibody reactivity pattern in serum of AID patients was analysed using a Luminex bead-based antigen array (SeroTag) and 1,600-8000 selected human protein antigens. We screened over 3000 serum samples from Sjögren's Syndrome $\quad(n=350), \quad$ SLE $\quad(n \geq 1000), \quad$ SSc $\quad(n \geq 250), \quad$ RA $(n \geq 1000)$, and several other AIDs and over 1000 healthy individuals to confirm known and to discover novel autoantibodies, create reduced autoantibody panels for differential diagnosis and disease subgrouping.

Results Apart from clear confirmation of the known benchmark autoantigens known for many years we have discovered over 80 novel autoantibodies, which were detected in frequencies of 10 to $>25 \%$ in selected AIDs. Some novel autoantibodies are specific for certain diseases, such as the major vault protein in SLE or BICD2 in SSc. Others are present in several diseases, indicating overlap syndromes. Multiplex panels of 50-100 AABs were generated and tested to allow for a subgroup definition of Sjögren's, SLE, and for clear segregation of SjS/SLE overlap syndrome patients. As well, subgrouping of SSc and early RA patients was achieved.

Conclusions A set of 100-150 autoantigens, half of them well established, the other half novel, succeed in differential diagnosis of AID, in some diseases already at early disease stage. This panel has been used in several drug trials to subgroup SLE, Sjögrens or RA into subgroups. Especially in SLE, outliers in the range of $10 \%-15 \%$ of the trial population were seen which can be used to curate a trial population, eventually to arrive at a more precise assessment of trials primary objectives.

\section{PS1:7 ANTINUCLEAR ANTIBODY (ANA) AND ANTINEUTROPHIL CYTOPLASMIC ANTIBODY TESTING IN A TERTIARY HEALTH ENTRE IN SHERBROOKE: AN ASSESSMENT OF THE ADHERENCE TO THE GUIDELINES AND THE IMPACTS ON THE DIAGNOSIS AND HEALTH CARE SYSTEM}

M Parfenova. Sherbrooke University, Sherbrooke, Canada

10.1136/lupus-2018-abstract.56

Objectives To describe antinuclear antibodies (ANA) and subserology ordering practices and to determine if its' indications meet the recommendations for ANA testing at the Sherbrooke University Health Centre, in Canada. To describe antineutrophil cytoplasmic antibodies (ANCA) practices and determine if they meet the 1999 guidelines proposed for ANCA testing, at the same centre.

Methods We identified the indications for the ANA and subserologies panel (Anti-SSA, anti-SSB, Anti-Jo1, Anti-Scl-70, Anti-Sm, Anti-U1 RNP) between 2012 and 2014 and compared to the guidelines for ANA testing. Moreover, the indications for ANCA tests were assessed and compared to the 1999 guidelines for the appropriate testing of ANCA.Variables included gender, age, ANA titer, subserologies panel, indication of ANA, ANCA, subtypes MPO and PR3, indication for ANCA, medical specialty, setting of the order and the final diagnosis.

Results There were a total of 268 ANA tests included. In $35.8 \%$ of cases $(n=96)$, ANA was ordered as per recommandations versus $63.8 \%$ of cases $(n=171)$ without indications. There were 104 subserologies ordered and 55.8\% were 